\title{
Resource Allocation Based on LSMT Architecture Perspective
}

\author{
Chao Liu ${ }^{1, \text { a }}$, Juanli Lan ${ }^{1, \mathrm{~b}}$, Honghong Zhang ${ }^{1, \mathrm{c}}$, Xiaoxue $\mathrm{Du}^{1, \mathrm{~d}}$, \\ International Business School, Shaanxi Normal University, 710000 \\ a1010273520@qq.com, ${ }^{b}$ lanjuanlimutou@163.com, ${ }^{\mathrm{c}} 1677863559 @$ qq.com, ${ }^{\mathrm{d}}$ xiaoxue950421@163.com
}

\begin{abstract}
Resource allocation is the core of the strategic management of companies, the allocation of appropriate resources to the most needed parts at the right time to realize optimal allocation of resources becomes an important aspect of enterprises' concern. This paper starts with the enterprise resource allocation process, analyzes the matching degree of LSTM model and resource allocation characteristics, as well as the maturity of LSMT model, and analyzes the feasibility of the application, so as to provide a new perspective for the future resource allocation management.
\end{abstract}

Keywords-Strategy management; Resource allocation; LSTM; RNN

\section{INTRODUCTION}

Resource allocation is an important part of enterprise strategic management, but the resources of the organization is not always is in a state of sufficient, or even if the total amount of resources is sufficient, its distribution in all divisions and departments are not always harmonious. The organizational structure and the limited funds for protection measures oblige enterprises to prioritize the most efficient and effective risk reduction schemes. Thus, decision makers need to know "which region should risk reduction focus on?" or alternatively, "where are the risk exposure hotspots located?" As a result, a sound understanding of rational allocation of resources is essential for the effective and efficient implementation of organization management. Therefore, grasp the method of resource management in the condition of limited organization resources seems to be a way of life for the foreseeable future. But so far, the study of the allocation of resources in enterprise strategy is less, most of the deviation from the actual needs, and the applicable scope is small, especially in the financial aspects of the allocation of resources research. Based on the perspective of literature review, this paper attempts to review and analyze relevant research results on resource allocation at home and abroad, and absorb excellent research methods and achievements. On the other hand, we hope to make up for the defects and deficiencies in the research by applying new research methods, so as to find new horizons for further research in the future.

\section{LITERATURE REVIEW}

Allocation of resources refers to the organizations based on strategic period is engaged in the business field, and establish the competitive advantage, its master of various economic resources, distribution on the quality and quantity, the purpose is to form strategic business structure or strategic system you need. In Bower's book, Managing the Resource Allocation Process (Bower, 1970), he proposes that the resource allocation process is decentralized in the hierarchical organization of various business departments and management companies. Mintzberg thinks that the process of the realization of the strategy is essentially the allocation of resources, only to the allocation of resources into consideration all the plan, calculation and analysis to be meaningful, most scholars agree with this view (Chandler, 1962:13, Ansoff, 1965:218),but in practice, the emergence of the agency relationship (Meckling,1976) causes the operator to be inconsistent with the owner's goal, operators focus more on short-term benefits than on strategic consideration of resource allocation (Bower, 1970'Bower \& Gilbert,2007),resource utility is greatly reduced, therefore, attracting the attention of most scholars. Up to now, scholarship on resource allocation falls primarily into one of three categories: studies of the processes by which resources are allocated in a firm (the emphasis of this paper), research on corporate capital allocation to divisions in a multibusiness firm, and work that examines factors affecting specific types of resource allocation. Bower proposed Bower-Burgelman model for organizational resource allocation process (Bower,1970), and Researchers in the relevant field have affirmed the above model (e.g., Ackerman,1970;see also Bower \& Doz, 1979, for a summary) and development (Burgelman,1983).At present, there are two main problems: firstly, although there are many researches on resource allocation, there is little research on enterprise strategy (Maritan \& Lee, 2017).The second is that the completed research content deviates from the actual needs, and the applicability is not high (Bower, 2017).To solve these problems, we propose an approach of spatial cluster analysis based on the aggregation of point data with respect to different spatial units. In the integration model, we focus on exposure and dedicated to analysis the organization risk events in time and space distribution, explore the environments of different regions of the comprehensive factor through data construction. Model comparison experiment is used to establish an optimal model for spatio-temporal prediction of risk events, and realize the analysis and prediction of risk events, and therefore provide 
quantitative analysis and forecast results for enterprises, especially trans-regional organizations, achieve the best risk prevention and control objectives with optimal resource efficiency.

\section{FEASIBILITY ANALYSIS}

Long short term memory(LSTM) is a kind of time circulating neural network, which was first developed by Hochreiter (Bengio et al,2013,Hochreiter \& Schmidhuber, 1997), it is an improvement on recurrent neural networks (RNN) ( Gers, Schraudolph, \& Schmidhuber, 2002), mainly solved the vanishing/exploding gradients problem( Bengio, Simard, \& Frasconi, 1994 ) of ANN, and improved by Alex Graves and promotion. After that, Alex Graves made an underwent improvement and promotion. The process of the .LSTM model is shown in Figure 1.

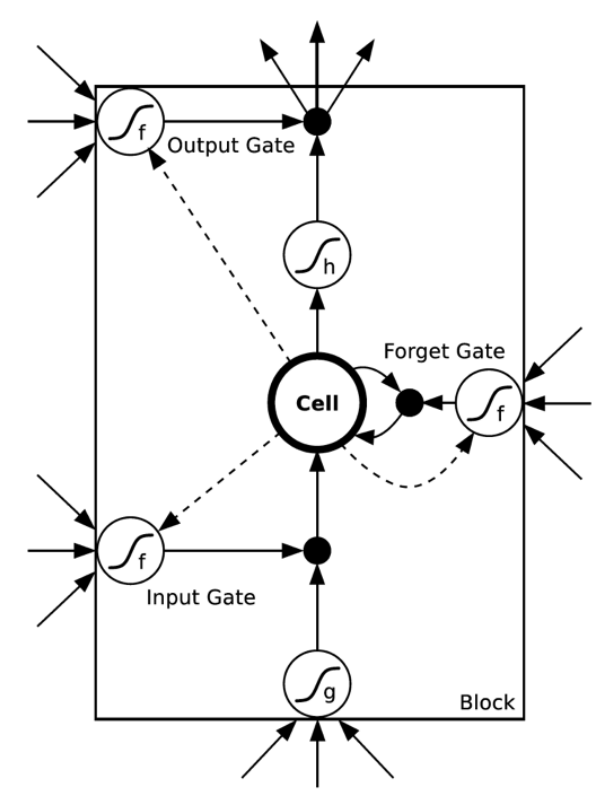

Fig. 1 LSTM model

The LSTM model structure consists of a set of interconnected recursive subnetworks, ie memory modules. Each network module contains one or more auto correlated memory nodes and three proliferation units - input, output, and oblivion gates. Writes, reads, and previous state resets for the data sequence. As shown in Figure 3-1, the valve node's calculation rule is: the sigmoid function takes the network's memory state as the input value; specifies a threshold, and if the output result reaches the threshold, the output value of the valve is compared with the calculation result of the current layer. Multiplies and multiplies the result of the multiplication as the input value of the next layer; if it does not reach the threshold, the output is discarded, ie, the forgotten rule has effect. All three gates use a differentiable sigmoid function, which guarantees that the three gates are trained to get the best parameters. The three doors symbolize the gates of information. They control the transmission of neuron information, how much new information is distributed to the current neuron, and how much of the current neuron's information is assigned to the next neuron. All three gates are nonlinear summation units.
They contain the activation function inside and outside the memory block and control the activation function of the unit through multiplication operations.

\section{A. From the characteristics of resource allocation}

There is an important component of the enterprise cost structure --- risk cost, risk cost refers to the cost and anticipated economic benefits that people have to spend after the occurrence of risk and the occurrence of risk accidents. Leading to cost risk events we call risk events, mainly include: the damage to or loss of property, fire, etc., due to such incidents are sudden and uncertainty, therefore it often leads to high risk cost of enterprises. However, according to the principle of causality, continuity and similarity (Ma Zhiyi, 1987), the development of things has certain internal correlations. How to find internal connections between events through big data, find patterns, predict beforehand, allocate resources ahead of time to prevent them, and then reduce the actual cost of risk loss should be paid more attention to in resource allocation. LSMT. It happens to have such a function. As LSTM introduces memory modules, it has the memory and processing functions of big data, so the calculated results are more objective and realistic. At the same time, since the LSTM has a selective switch at the gate of forgetting (as shown in Figure 2).

The black solid circle in the figure represents the information transfer, and the node's calculation result value will continue to be passed to the next layer or the next time point. ; The cross sign indicates the shielding of the information, the node's calculation result is not input to the next layer or the next time point. When the valve is opened, the training results of the preceding neuron will affect the calculation of the current neuron, and when the valve is closed, the information of the preceding neuron will not affect the calculation of the current neuron. Therefore, through the adjustment of the valve switch, we can achieve the impact of historical time series on the late neurons. If we do not want the information from the previous moment to affect the subsequent data, such as the analysis of new passages or chapters in natural language processing, then simply turning off the valve can stop the transmission of information. The LSTM network influences each other by opening the valve and closing the valve control sequence. Therefore, we believe that LSTM has the ability to meet the needs of regulating enterprise resource allocation. 


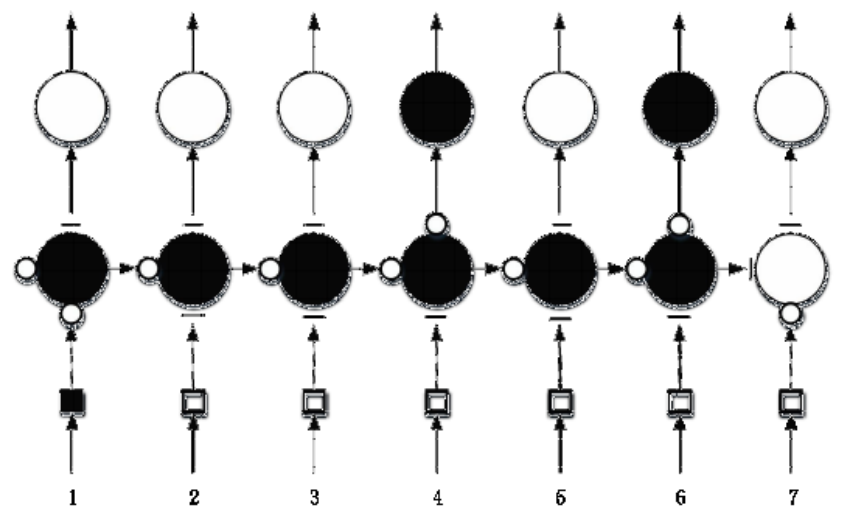

Fig. 2 LSTM can avoid gradient disappearance

\section{B. Flexibility of LSMT}

Firstly, we start with the processing power of the data, LSTM can make a selective memory of the input data via it's memory cells and gate units (Hochreiter \& Schmidhuber, 1997), solving the vanishing/exploding gradients problem (Bengio, Simard, \& Frasconi, 1994) of ANN [1]; secondly, it can handle up to several million or more, as much as most to a few millions or more. Finally, according to the different requirements of the researcher, LSTM can be combined with various methods, Corcoran, Wilson and Ware (2003) introduced a method of forecasting crime and disorder related to geographic areas using the combination of autoregressive models and ANN to identify hot spots; Yu jia bin, Shang fang and other scholars used gf-lstm network model and realized the prediction of algae bloom (2018) [2]. Xiaolei Ma, Zhimin Tao and some other scholars (2017) successfully used Long ShortTerm Neural Network (LSTM NN), to capture nonlinear traffic dynamic in an effective manner. Some scholars (Yu Zhao, Rennong Yanga, et al, 2018) combined bidirectional long short-term memory (BLSTM) and mixture density network (MDN) approach, and it torned out to be better models in terms of the convergence speed and accuracy.

\section{The maturity of LSMT}

From emergence to development, LSTM has shown excellent performance in a variety of applications such as natural language processing, speech recognition, and computer vision over recurrent neural network (DONG WU, MINGMIN CHI,2017),text analysis, and many others (LeCun et al., 2015).

For example, Xue and Brown (2003) developed a decision model for spatial site selection by criminals. Liao, Wang, Xia, and Hannam (2012) studied environmental emergency events. Galbrun, Pelechrinis, and Terzi (2016) studied crime events in cities. Yang Xunzheng as well as other three scholars analysed pollution emission of generator sets,(2016), they solved the problem that the traditional least square method and machine learning method are difficult to extract the valid features from the data of generator sets by using the LSTM architecture [3]. Shan Shaojie, Li Hanjun (2017) made a comparison experiment and showed that the prediction method based on LSTM model is superior to SVM and MLP in prediction accuracy and generalization ability [4]. Several scholars use LSTM model to improve the prediction accuracy of emergency response systems (Bitzel Cortez, Berny Carrera, Young-Jin Kim\&Jae-Yoon Jung, 2018).Li Zelong and Yang Chunjie (2018) introduced LSTM-RNN model into the field of chemical industry, by using the new method they improved the accuracy and stationarity of ferrosilicon content [5]. Dai Jiejie and Song Hui (2018) utilized the LSTM prediction model to predict the transformer status after 1 week, the accuracy rate was $94.4 \%$, and $81.2 \%$ after 1 month [6];Yu Jiahong,Bao Zhejing(2018) proposed LSTM method, Under the premise of the given precision, the global optimum of user load is realized, and the computation amount is reduced significantly[7];Shikhar Srivastava and Stefan Lessmann(2018) made a Comparative Study on solar energy and concluded that LSTM outperforms a large number of alternative methods with substantial margin and an average forecast skill of $52.2 \%$ over the persistence model.

\section{RESEARCH REVIEW AND PROSPECT}

Since LSTM was proposed (Hochreiter \& Schmidhuber, 1997), the LSTM model has been applied in many fields and has been applied in a wide range of applications through the combination of various methods such as various generalized autoregressive conditional heteroscedasticity (GARCH)-type models (Ha Young Kim \& Chang Hyun Won,2018),solving a lot of problems. However, so far, LSMT's applications have been mainly focused on the computer and other fields, which involve less enterprise level. This paper is based on the theory level and committed to applying the application to enterprise resource allocation. However, with narrow aspect consideration, and no substantial data to support, there still exists a large gap and improvement to apply LSTM at the enterprise level.

\section{ACKNOWLEDGMENT}

This research was financially supported by Xi'an Social Science Planning Fund Project (18J161) and China Postdoctoral Science Fund Project (2018M633446).

\section{REFERENCES}

[1] Bitzel Cortez, Berny Carrera, Young-Jin Kim, Jae-Yoon Jung An.

[2] Architecture for emergency event prediction using LSTM recurrent neural networks [J]. Expert Systems With Applications 97 (2018) 315324.

[3] Yu Jiabin, Shang Fangfang, Wang Xiaoyi, Xu Jiping, Wang Li, Zhang Huixi, Zheng Lei. Blue algae bloom prediction method based on GFLSTM network [J/OL]. Computer application: 1-6[2018-06-14].(In Chinese)

[4] Yang Xunzheng,Ke Yuyang,Liang Xiao,Xiong Yan.Study on Prediction of Pollutant Emission of Generator Based on LSTM[J]. Electrical Automation,2016,38(05):22-25.(In Chinese)

[5] Shan Shaojie, Li Hanjun, Wang Wei, Tang Xiaoying. Prediction of single-lead EEG seizures based on LSTM model [J/OL]. Computer Application Research, 2018(11):1-6[2018-06-14]. (In Chinese)

[6] Li Zelong,Yang Chunjie,Liu Wenhui,Zhou Heng,Li Yuxuan.Prediction of Silicon Content in Molten Iron Based on LSTM-RNN Model [J]. Journal of Chemical Industry and Engineering(China), 2018,69(03):992997.(In Chinese)

[7] Dai Jiejie,Song Hui,Sheng Gejun,Jiang Xiuchen,Wang Jianyi,Chen Yufeng.Study on Prediction Method of Power Transformer Operation State Using LSTM Network [J]. High Voltage Engineering,2018,44(04):1099-1106. (In Chinese)

[8] Yu Jiahong,Bao Zhejing,Li Zhijie.User Load Interval Prediction Method Based on LSTM[J].Industrial Control Computer,2018,31(04):100-102. (In Chinese) 